Furness, G. \& Gaze, H. W. (1952). J. gen. Microbiol. 6, 149

\title{
A Method of Preparing Individual Sterile Pipettes
}

\author{
By G. FURNESS AND H. W. GAZE \\ From the Department of Bacteriology, University of Manchester
}

SUMMARY: Tubes of Kraft paper which can be used repeatedly up to six times are used for encasing individual pipettes.

The laboratory practice of wrapping pipettes individually in paper before sterilization has a number of disadvantages. The process is time-consuming, when thin paper is used there is a danger that it may crack and lead to contamination of the pipette, and tough Kraft paper is expensive and scarce. To overcome these difficulties seamless tubes of Kraft paper sufficiently thick to be rigid were obtained from the Star Paper Tube Co. Ltd., Albert Mills, Hollinwood, Oldham. The tubes are $18 \mathrm{in.} \mathrm{long,} \mathrm{to} \mathrm{allow} \mathrm{the} \mathrm{end} \mathrm{to} \mathrm{be} \mathrm{trimmed,}$ and of $\frac{3}{8} \mathrm{in}$. diameter for pipettes under $5 \mathrm{ml}$. and $\frac{1}{2}$ in. diameter for those of 5 and $10 \mathrm{ml}$. capacity; other sizes can be obtained.

Before use one end of the tube is plugged with cotton-wool which is retained in position by stapling with an ordinary office stapler so that this end of the tube is pinched flat with the cotton-wool retained inside. After inserting the pipette the other end of the tube is plugged as for test tubes, different sizes of pipette being identified by differently coloured wools.

The encased pipettes are sterilized in the hot air oven at $160^{\circ}$ for $1 \mathrm{hr}$. The durability of the tubes is such that they can be heated six times at $180^{\circ}$ for $1 \mathrm{hr}$. before they become brittle and crack. They should, however, be inspected each time before use to ensure that they are in a satisfactory condition. The open end of the tube will withstand moderate flaming and can be trimmed if it should become charred.

This method has the following advantages over wrapping. Assuming an average of six sterilizations, the cost of materials is halved, the time required in preparation is about one-quarter and much tedious repetitive work obviated. The cotton-wool and rigidity of the case protect the pipette from accidental damage and allow it to be more easily extracted than from paper wrappers. The procedure has been in routine use in this laboratory for some time and has given general satisfaction.

(Received 7 August 1951) 